\title{
Manejo de la glicemia en el contexto agudo del evento cerebrovascular isquémico.
}

Glycemic management in the acute ischemic stroke. Dr. Lorenzo Marín Barboza ${ }^{1}$ Dr. Daniel Hernández Durán ${ }^{2}$ Dr. Douglas Mejías Anchía ${ }^{3}$

1,2 y 3 Médico general. Trabajador independiente, San José Costa Rica.

Contacto: marinlore@gmail.com

\section{Resumen}

La enfermedad Cerebrovascular es una de las principales causas de morbimortalidad a nivel global, siendo el evento isquémico, donde el flujo sanguíneo hacia el cerebro se interrumpe, la forma de presentación más común, abarcando más de un $80 \%$ de los eventos cerebrovasculares (1).

Los Eventos Cerebrovasculares Isquémicos (ECVI) se acompañan de cifras glucémicas elevadas en un $40 \%$ de las ocasiones, ya sean pacientes con un diagnóstico previo de diabetes o pacientes no diabéticos (2, 3). La cuantificación de estos valores, detectables desde el ingreso, es esencial, ya que es conocido que la glucemia en la fase aguda del evento se comporta como un predictor independiente de resultados negativos en la evolución del paciente. Aun así, poco se sabe, con evidencia de peso, de cuál es el rango glucémico ideal en el que se debe mantener a estos pacientes para prevenir estas complicaciones y mucho menos se tiene claro cuál es la manera más eficaz y oportuna de controlar estos parámetros metabólicos. Por eso, el objetivo de este documento es revisar la bibliografía reciente que trate este tópico, con el fin de recabar la información necesaria que pueda esclarecer el manejo de la hiperglucemia en el contexto del ECVI, e indagar en la eficacia y seguridad de los métodos actualmente en uso.

Palabras clave:

Evento cerebrovascular; Isquemia cerebral; hiperglicemia; glúcemia

\section{Abstract}

The cerebrovascular disease is one of the mayor causes of morbidity and mortality globally, being the ischemic event, where the cerebral blood flow is interrupted, the most common presentation comprising an $68 \%$ of all strokes, and $32 \%$ hemorrhagic strokes.

The ischemic strokes, are accompanied in $40 \%$ of times by elevated values of glucose, be it in patients with a previous diagnosi of diabetes or not known diabetics. The quantification

Recibido: $08 / \mathrm{jul} / 2020$

Aceptado: 12/nov/2020

Publicado: 15/dic/2020 of these values, detectable from the admission is essential, since it is known that the glycemia in the acute phase behaves like an independent predictor of negative patient's evolution outcomes. Still, little is known with strong evidence, about the ideal glycemic range for these patients to prevent complications, and less clear is the most effective way to control these metabolic parameters. That is why the objective of this document is to review the recent bibliography on this issue, in order to gather the necessary information to clarify the management of hyperglycemia in the context of ischemic stroke and inquire in the efficacy and safety of the methods on use now.

Keywords:

Stroke; Brain ischemia; Hyperglycemia; Blood glucose. 
Introducción

El evento cerebrovascular isquémico (ECVI) es una de las principales causas de muerte y discapacidad en el mundo, razón por la que requiere un abordaje diagnóstico y terapéutico expedito. La definición de clínica de estos eventos varía constantemente en función del avance de las neuroimágenes, así también hay una actualización constante de las guías de manejo inicial y de sus complicaciones concomitantes (4). El abordaje integral de un evento agudo debe ser realizado en un escenario ideal, donde se cuente con una unidad de atención al paciente con ECVI, conformado por un equipo interdisciplinario de médicos especialistas, enfermeros capacitados y otros profesionales clínicos, que al mismo tiempo manejan una base de conocimiento actualizado en el manejo clínico y que formulen un protocolo regional para el manejo del ECVI(5).

La hiperglicemia es un hallazgo común en el ECVI, se puede encontrar tanto en pacientes diabéticos como en no diabéticos y puede ser identificada desde el ingreso o durante la estancia hospitalaria. Se destaca su importancia puesto que se ha asociado con peores resultados funcionales $(2,5,6)$. La eficacia del manejo glicémico intensivo ha permanecido desconocida, pero recientemente se ha actualizado la evidencia al respecto. Con esto se genera una apertura al panorama de la euglicemia. Por estas razones, es evidente la importancia de una revisión de la literatura reciente sobre la terapéutica de la hiperglicemia en el paciente con ECVI y su relevancia en el cambio de la práctica clínica, además se revisan las recomendaciones previas sobre este tópico.

\section{La relación del ECVI y la hiperglicemia}

El ECVI se define como un episodio de disfunción neurológica causado por un infarto focal cerebral, espinal o retinal (7); en el abordaje inicial es muy difícil saber clínicamente si se trata de un ECVI, de tipo hemorrágico o el calibre del vaso comprometido (8); por lo tanto, una de las metas principales es diagnosticar la naturaleza del evento, utilizando imágenes médicas y descartar que se trate de una deficiencia neurológica secundaria a hipoglicemia (5). Una vez confirmado el ECVI, se determina si es candidato a trombólisis; la evolución de dicho manejo ha sido un reto (9). Es válido recalcar que la glicemia es el único parámetro que debe ser medido en todos los pacientes previo a la infusión de alteplasa, ya que al ser un parámetro que es de rápido acceso, fácilmente se pueden descartar dos situaciones que pueden mimetizar un ECVI, las cuales son la hiperglicemia y la hipoglicemia (5). Posteriormente monitoreo hospitalario, manejo de comorbilidades y rehabilitación; en caso de ser necesario un manejo paliativo, incluso se menciona que la glucemia es un factor incluido en modelos de valor predictivo para el ECVI (10). En el contexto agudo también están los elementos fundamentales como la historia clínica, el examen físico con inspección neurológica rápida, paralelamente el manejo de la vía aérea, patrón respiratorio, estado circulatorio y su debida estabilización médica en caso de requerir; además el paciente con evento cerebro vascular frecuentemente presenta diversos acompañantes que requieren atención e intervenciones a corto mediano que logran disminuir resultados negativos: manejo adecuado de la presión arterial, gestión del agotamiento de volumen y medio interno, hiperglicemia, fiebre.

La hiperglucemia, generalmente definida como un nivel de glucemia $126 \mathrm{mg} / \mathrm{dL}$, es una complicación frecuente que afecta hasta a dos tercios de todos los pacientes, y está asociado con alteración de la recanalización, disminución de la perfusión, aumento de lesión por reperfusión y se asocia con peores pronósticos (11-13). Es importante precisar sobre la hiperglicemia por estrés donde es activado el eje hipotalámico-pituitario-adrenal y el sistema nervioso simpático, generando una respuesta de liberación de liberación de hormonas del estrés como catecolaminas y cortisol, lo que estimula la producción de glucosa por glucogenólisis, gluconeogénesis, proteólisis y lipólisis, además el aumento en la epinefrina podría contribuir con una resistencia periférica y transitoria a la insulina $(2,3)$. Sin embargo, sobresale que aunque muchos de estos pacientes no son diabéticos conocidos, en muchos será la primera manifestación de una enfermedad crónica aún no diagnosticada. En estos casos es difícil diferenciar si se trata de una hiperglicemia por estrés del proceso agudo o una hiperglicemia en un paciente diabético aún no diagnosticado, donde entraría a jugar un papel importante la hemoglobina glicosilada dando un panorama cronológico más amplio.

En el ECVI, el estrés fisiológico de la enfermedad aguda lleva a un estado hipermetabólico ya descrito, en el cual se activa la cascada inflamatoria dando lugar a mayor daño celular con elevación del lactato y formación de radicales libres. Como consecuencia del aumento de concentración de glutamato secundaria a hiperglicemia, una cantidad excesiva de calcio en el flujo ocurre a través de canales iónicos resultantes en lesión mitocondrial y muerte celular. Aunque la hiperglicemia por estrés no parece estar asociada directamente con el resultado del ECVI, dado que los pacientes con esta condición tienen más prevalencia de factores de riesgo cardiovascular que los pacientes con normoglicemia, se requieren más estudios para validar dichos hallazgos (13). 


\section{Complicaciones de la hiperglicemia en ECVI}

Como se mencionó previamente, la hiperglicemia en el ECVI está asociada a mayores complicaciones, peores pronósticos e inclusive disminuye los beneficios de los resultados posteriores a la terapia con tPA $(13,14)$. En un estudio reciente, se recopiló la información de 58265 pacientes con ECVI de 1.408 centros que recibieron tPA entre los años 2009 y 2013 en la base de datos Get With The Guidelines Stroke (GWTG-Stroke). Una glicemia > $140 \mathrm{mg} / \mathrm{dL}$ y HbA1c $>6.5$ se asociaron con peores resultados clínicos (hemorragia intracraneal sintomática, hemorragia potencialmente mortal, mortalidad hospitalaria y duración de la estancia) en pacientes diabéticos y no diabéticos(14). En otro estudio donde se pretendía asociar una hiperglicemia con el deterioro neurológico y con el volumen de crecimiento del infarto. Se estudiaron a 375 pacientes con accidente cerebrovascular isquémico agudo con oclusión arterial dentro de las 24 horas del inicio. La diabetes fue diagnosticada en pacientes con antecedentes conocidos de diabetes o valor de $\mathrm{HbA} 1 \mathrm{c} \geq 6.5 \%$. El volumen del infarto se midió al ingreso y en el seguimiento dentro de las 48 horas con imágenes ponderadas por difusión. Deterioro neurológico se definió como un aumento de $\geq 4$ puntos, según los Institutos Nacionales de Salud Stroke Scale, puntuación dentro de los 7 días posteriores al inicio del accidente cerebrovascular. En este análisis se demostró que el nivel elevado de glucemia se asocia independientemente con crecimiento del volumen del infarto en todos los pacientes aún sin diabetes conocida previamente, pero no así en los ya diagnosticados con diabetes crónicamente. Además, el nivel elevado de glucemia fue independientemente asociado con deterioro neurológico en todos los pacientes (3). En otra investigación se logra demostrar que la hiperglicemia se asocia a una mayor incidencia de infecciones posterior al ECVI. En el estudio se definió infección posterior al accidente cerebrovascular como cualquier infección durante la admisión juzgada por un comité experto. De 1.676 pacientes con accidente cerebrovascular isquémico no diabético, 338 (20\%) tenían hiperglucemia de admisión. Después del ajuste por posibles variables de confusión, la hiperglucemia de ingreso se asoció con infección posterior al accidente cerebrovascular (odds ratio ajustado [aOR] 2.31, IC 95\% 1.31-4.07), peor funcional a los 3 meses resultado (aOR 1.40 común, IC 95\% 1.12-1.73) y mortalidad a los 3 meses (aOR 2.11, IC 95\% 1.40-3.19) (12).

Sobre las recomendaciones previas en manejo de la hiperglicemia

Las guías actuales de la American Heart Association/American Stroke Association

(AHA/ASA) sugieren con evidencia limitada, tratar la hiperglicemia para lograr una meta entre los $140-180 \mathrm{mg} / \mathrm{dl}$. Además, recomiendan que una infusión de insulina se puede utilizar para evitar hiperglucemia significativa y evitar la hipoglicemia en todo momento $(5,15)$.

Recientemente se publicaron los resultados del ensayo clínico randomizado Stroke Hyperglycemia Insulin Network Effort (SHINE), el cual evaluó el control glucémico intensivo versus el estándar de la hiperglicemia en pacientes con ECVI; la hipótesis era que el control intensivo de la glucemia iba a mejorar los resultados funcionales en pacientes con ECVI e hiperglicemia, en comparación con control estándar $(6,16)$.

\section{Sobre la nueva evidencia: el ensayo clínico SHINE $(6,16)$}

Este ensayo incluyó 1151 pacientes adultos con hiperglicemia y no más de 12 horas posteriores al inicio de los síntomas de ECVI agudo, con un puntaje en el National Institutes of Health Stroke Scale (NIHSS) score de 3 a 22 en 63 centros, del 2012 al 2018. La hiperglicemia se definió como glicemia al momento de la inscripción $>110 \mathrm{mg} / \mathrm{dl}$ en pacientes con diabetes mellitus (DM) tipo 2 y $>140 \mathrm{mg} / \mathrm{dl}$ en pacientes sin DM conocida.

Se excluyeron aquellos con DM tipo 1, diálisis renal e indicación clínica de terapia de infusión continua de insulina intravenosa.

Al grupo de control intensivo se intervino con infusión intravenosa de insulina necesaria para mantener una concentración de glucemia entre 80-130 mg /dl; el grupo de control estándar recibió insulina subcutánea con escala variable que fue administrada cada 6 horas según fue necesario para mantener una concentración de glucemia de 80-179 mg/dl; si en 24 a 48 horas no se lograba alcanzar la meta, la dosis era incrementada, incluida la basal. El período de tratamiento fue de 72 horas. El resultado primario de eficacia fue un resultado favorable a los 90 días después de aleatorización. Un resultado favorable fue definido por un puntaje en la escala modificada de ranking de 0 en pacientes con un puntaje basal de 3 a 7 en el NIHSS, un puntaje en la escala modificada de ranking de 0 a 1 en pacientes con un puntaje basal de 8 a 14 en el NIHSS, un puntaje en la escala modificada de ranking de 0 a 2 en pacientes con un puntaje basal de 15 a 22 en el NIHSS. 
Hubo un resultado favorable en el $20 \%$ del grupo con terapia intensiva y en el $21 \%$ del grupo con terapia estándar (riesgo relativo ajustado, $0.97[95 \% \mathrm{Cl}, 0.87$ to 1.08$]$,

$\mathrm{P}=.5$ ). Aproximadamente, el $80 \%$ de ambos grupos tenía DM tipo 2 y $68 \%$ del total recibió terapia de reperfusión. El tratamiento se detuvo de forma temprana por hipoglicemia (glicemia $<40 \mathrm{mg} / \mathrm{dl}$ ) en el $11.2 \%$ del grupo con terapia intensiva y $3.2 \%$ del grupo con terapia estándar.

En comparación con el GIST-UK trial, SHINE es el único otro ensayo de eficacia aleatorizado para tratar la hiperglucemia durante el accidente cerebrovascular isquémico agudo realizado en 2007, donde la diferencia entre los niveles de glucemia, durante un período de tratamiento de 24 horas fue de solo $10 \mathrm{mg} / \mathrm{dL}(0.6 \mathrm{mmol} / \mathrm{L})$.

El estudio logró demostrar una marcada diferencia en la glicemia promedio de ambos grupos (diferencia de $61 \mathrm{mg} / \mathrm{dl}$ ), $\sin$ embargo no hubo diferencia significativa en el resultado primario de eficacia y la terapia intensiva no mejoró los resultados funcionales comparados con la terapia estándar. Por lo tanto, estos datos no dan soporte al uso de control glucémico intensivo.

\section{Conclusiones}

El control glucémico en el paciente con ECVI es un aspecto de suma importancia en el abordaje integral de esta patología, siempre asociado a la atención oportuna y casi inmediata; y al tratamiento fibrinolítico. Se sabe con certeza que la hiperglicemia oscurece el pronóstico de pacientes con ECVI, sin embargo, no se han podido determinar metas claras para los niveles glicémicos en el contexto descrito. Los expertos sugieren un rango glicémico entre 140-180 mg/dl y monitorización constante para evitar la hipoglicemia que a su vez aumenta la incidencia de complicaciones y el riesgo de muerte complicando el manejo agudo de la patología. Sin embargo, aún no se ha logrado un consenso en la manera más efectiva para lograr este objetivo sugerido. A pesar de que la hipótesis de un manejo glucémico intensivo parecía una buena opción según las premisas, la evidencia no apoya su empleo al no demostrarse en estudios recientes, diferencia significativa en resultados con respecto al manejo estándar.

\section{REFERENCIAS BIBLIOGRÁFICAS}

1. Anrather J, Iadecola C. Inflammation and Stroke: An Overview. Neurotherapeutics. octubre de 2016;13(4):661-70.

2. Clark ME, Payton JE, Pittiglio LI. Acute Ischemic Stroke and Hyperglycemia: Crit Care Nurs Q. 2014;37(2):182-7.

3. Shimoyama T, Kimura K, Uemura J, Saji N, Shibazaki K. Elevated glucose level adversely affects infarct volume growth and neurological deterioration in non-diabetic stroke patients, but not diabetic stroke patients. Eur J Neurol. marzo de 2014;21(3):402-10.

4. Campbell BCV, De Silva DA, Macleod MR, Coutts SB, Schwamm LH, Davis SM, et al. Ischaemic stroke. Nat Rev Dis Primer. diciembre de 2019;5(1):70.

5. Powers WJ, Rabinstein AA, Ackerson T, Adeoye OM, Bambakidis NC, Becker K, et al. 2018 Guidelines for the Early Management of Patients With Acute Ischemic Stroke: A Guideline for Healthcare Professionals From the American Heart Association/American Stroke Association. Stroke [Internet]. marzo de 2018 [citado 26 de octubre de 2020];49(3). Disponible en: https://www.ahajournals.org/doi/10.1161/STR.0000000000000158

6. Johnston KC, Bruno A, Pauls Q, Hall CE, Barrett KM, Barsan W, et al. Intensive vs Standard Treatment of Hyperglycemia and Functional Outcome in Patients With Acute Ischemic Stroke: The SHINE Randomized Clinical Trial. JAMA. 23 de julio de 2019;322(4):326.

7. Sacco RL, Kasner SE, Broderick JP, Caplan LR, Connors JJ (Buddy), Culebras A, et al. An Updated Definition of Stroke for the 21st Century: A Statement for Healthcare Professionals From the American Heart Association/American Stroke Association. Stroke. julio de 2013;44(7):2064-89.

8. Smith EE, Kent DM, Bulsara KR, Leung LY, Lichtman JH, Reeves MJ, et al. Accuracy of Prediction Instruments for Diag-

2020 Marín Barboza et al. vol 4, número 6 Diciembre 2020 - Enero 2021 DOI: https://doi.org/10.34192/cienciaysalud.v4i6.112 
nosing Large Vessel Occlusion in Individuals With Suspected Stroke: A Systematic Review for the 2018 Guidelines for the Early Management of Patients With Acute Ischemic Stroke. Stroke [Internet]. marzo de 2018 [citado 26 de octubre de 2020];49(3). Disponible en: https://www.ahajournals.org/doi/10.1161/STR.0000000000000160

9. Fisher M, Saver JL. Future directions of acute ischaemic stroke therapy. Lancet Neurol. julio de 2015;14(7):758-67. 10. Holloway RG, Arnold RM, Creutzfeldt CJ, Lewis EF, Lutz BJ, McCann RM, et al. Palliative and End-of-Life Care in Stroke: A Statement for Healthcare Professionals From the American Heart Association/American Stroke Association. Stroke. junio de 2014;45(6):1887-916.

11. Fuentes B, Sanz-Cuesta BE, Gutiérrez-Fernández M, Martínez-Sánchez P, Lisbona A, Madero-Jarabo R, et al. Glycemia in Acute Stroke II study: a call to improve post-stroke hyperglycemia management in clinical practice. Eur J Neurol. septiembre de 2017;24(9):1091-8.

12. Zonneveld TP, Nederkoorn PJ, Westendorp WF, Brouwer MC, van de Beek D, Kruyt ND, et al. Hyperglycemia predicts poststroke infections in acute ischemic stroke. Neurology. 11 de abril de 2017;88(15):1415-21.

13. Tziomalos K, Dimitriou P, Bouziana SD, Spanou M, Kostaki S, Angelopoulou S-M, et al. Stress hyperglycemia and acute ischemic stroke in-hospital outcome. Metabolism. febrero de 2017;67:99-105.

14. Masrur S, Cox M, Bhatt DL, Smith EE, Ellrodt G, Fonarow GC, et al. Association of Acute and Chronic Hyperglycemia With Acute Ischemic Stroke Outcomes Post-Thrombolysis: Findings From Get With The Guidelines-Stroke. J Am Heart Assoc [Internet]. 27 de octubre de 2015 [citado 26 de octubre de 2020];4(10). Disponible en: https://www.ahajournals.org/ doi/10.1161/JAHA.115.002193

15. Wijdicks EFM, Sheth KN, Carter BS, Greer DM, Kasner SE, Kimberly WT, et al. Recommendations for the Management of Cerebral and Cerebellar Infarction With Swelling: A Statement for Healthcare Professionals From the American Heart Association/American Stroke Association. Stroke. abril de 2014;45(4):1222-38.

16. Bruno A, Durkalski VL, Hall CE, Juneja R, Barsan WG, Janis S, et al. The Stroke Hyperglycemia Insulin Network Effort (SHINE) Trial Protocol: A Randomized, Blinded, Efficacy Trial of Standard vs. Intensive Hyperglycemia Management in Acute Stroke. Int J Stroke. febrero de 2014;9(2):246-51. 